\title{
Validación de un modelo simulado inanimado basado en impresión 3D de ureterorrenoscopía flexible
}

\author{
Rodrigo Neira S. ${ }^{1}$ Julián Varas C. ${ }^{1}$ y Gastón Astroza E. ${ }^{1}$
}

\section{Validation of an inanimate simulated model based on flexible ureterorenoscopy $3 \mathrm{D}$ printing}

\begin{abstract}
Aim: To establish the face, content, and construction validity of a flexible ureterorenoscopy simulation program. Materials and Method: A simulation model for flexible ureterorenoscopy was developed using silicone on which eight colored marks were marked on the calyxes. For validation, expert urologists and residents with varying amounts of experience in this procedure were recruited. They were separated into three groups: 1) G1 for residents without experience in ureteroscopy; 2) G2 for residents with variable experience; and 3) G3 for expert urologists. They were asked to perform a full navigation of the model in a maximum time of $600 \mathrm{sec}$. At the end, each participant answered a survey regarding the usefulness and realistic nature of the model. In addition, total time, number of marks found, and times of re-entry to the calyxes were measured. Results: A median of 8.6 points was obtained for the utility of the model and 6.75 points for its realistic nature. The total navigation times were 504, 293, and 133 seconds for $\mathrm{G} 1, \mathrm{G} 2$, and G3, respectively $(\mathrm{p}=0.02)$. Of the eight marks, an average of 5.1, 6.6, and 7.3, $(\mathrm{p}=0.18)$ were found with an average of 9.5, 3.8, and 1.3 exploration reattempts at the chalices in the corresponding groups $(p=0.11)$. Conclusions: Face and content validity was established for this simulation model of flexible ureterorenoscopy. This flexible ureterorenoscopy simulation program allowed us to differentiate the level of expertise in terms of reduction in navigation time.

Key words: ureteroscopy; learning curve; clinical simulation; clinical competence.
\end{abstract}

\section{Resumen}

Objetivo: Establecer validez aparente, de contenido y constructo, de un programa de simulación de ureterorrenoscopía flexible. Materiales y Método: Se desarrolló un modelo de simulación de silicona para ureterorrenoscopía flexible, en el cual se establecieron 8 marcas de colores en los distintos cálices. Para la validación, se reclutaron urólogos expertos y residentes de urología con experiencia variable en este procedimiento. Se separaron en 3 grupos: G1 para residentes sin experiencia en ureteroscopía, G2 para residentes con experiencia variable y G3 para urólogos expertos. Se les solicitó realizar una navegación completa del modelo, en un tiempo máximo de 600 segundos. Al finalizar, cada participante contestó una encuesta respecto a la utilidad y realismo del modelo. Además, se midió tiempo total, número de puntos encontrados y cantidad de veces de reingreso a los cálices para validación de constructo. Resultados: 15 personas participaron en la evaluación. Se obtuvo una mediana de 8,6 puntos para la utilidad del modelo y 6,75 puntos para el realismo de este. Los tiempos totales de navegación fueron 504, 293 y 133 segundos para G1, G2 y G3 respectivamente $(\mathrm{p}=0,02)$. De las 8 marcas, se encontraron en promedio 5,1, 6,6 y 7,3 $(\mathrm{p}=0,18)$, presentando un promedio de 9,5, 3,8 y 1,3 reintentos de exploración de los cálices en los respectivos grupos $(\mathrm{p}=0,11)$. Conclusiones: Se establece validez aparente y contenido para un modelo de ureterorrenoscopía flexible. El programa de simulación de ureterorrenoscopía flexible establecido, permite diferenciar novatos de expertos en cuanto a reducción en los tiempos de navegación.

Palabras clave: ureteroscopía; curva de aprendizaje; simulación clínica; competencia clínica.
Facultad de Medicina, Pontificia Universidad Católica de Chile. Santiago, Chile.

Recibido el 21 de agosto de 2019 y aceptado para publicación el 2 de enero de 2020

Correspondencia a: Dr. Rodrigo Neira S r.neirasoro@gmail.com 


\section{Introducción}

La simulación quirúrgica es una herramienta que en los últimos años se ha convertido en una pieza importante en el entrenamiento de los cirujanos, tanto para la adquisición de habilidades técnicas como no técnicas, permitiéndonos acortar la curva de aprendizaje en un ambiente seguro y reproducible ${ }^{1-3}$.

Debido a la creciente complejidad de los procedimientos y al desarrollo de nuevas tecnologías en el ámbito quirúrgico, se hace necesario encontrar herramientas que nos permitan adquirir las destrezas necesarias en ambientes seguros. En este contexto, los laboratorios de simulación han adquirido una creciente importancia en la formación médica ${ }^{1,2}$. La formación urológica basada en simulación (así como la de otras especialidades quirúrgicas) es un ejemplo de esta realidad, siendo necesario que se lleve a cabo utilizando programas de entrenamiento que se encuentren debidamente validados ${ }^{1,4}$.

La ureterorrenoscopía flexible es una técnica ampliamente utilizada en el manejo de litiasis urinaria proximal, así como una herramienta diagnóstica frente a sospecha de tumores de urotelio alto. Debido a la fragilidad de los ureterorrenoscopios, el alto costo asociado a estos insumos y la necesidad de conocer su orientación espacial para la correcta realización de este procedimiento, se hace necesario establecer un programa de formación para el desarrollo de habilidades técnicas.

El objetivo de este estudio consiste en establecer validez aparente, de contenido y constructo de un programa de entrenamiento en ureterorrenoscopía flexible, utilizando un modelo de simulación inanimado de bajo costo desarrollado en nuestra institución, como una herramienta para la adquisición de habilidades en endourología por parte de los residentes de urología.

\section{Materiales y Método}

\section{Desarrollo del modelo}

Se desarrolló un modelo de simulación de ureterorrenoscopía flexible a partir de la impresión 3D de una estructura de silicona de un riñón derecho con su respectivo uréter y sistema colector, basándose en la urografía por tomografía axial computada (Uro-Tac) de un paciente portador de urolitiasis. A nivel ureteral, se instaló una vaina de acceso ureteral de 35 centímetros de largo y 12-14 French de diámetro interno-externo respectivamente. Se realizaron 8 marcas de distintos colores en los diferentes cálices: 3 de ellos en el cáliz superior, 2 en el cáliz medio y 3 en el inferior. El costo de confección de la matriz fue de aproximadamente USD $\$ 100$, y reproducir cada copia del modelo tiene un costo de USD $\$ 30$.

Se preparó el modelo llenando el sistema colector con solución salina $0,9 \%$. En la Figura 1 se muestra la anatomía del modelo y la visión obtenida mediante ureteroscopía.

\section{Validación del modelo}

Una vez obtenida la aprobación por el comité de ética de nuestra institución, para la validación, se reclutó residentes de urología con experiencia variable en ureteroscopía flexible, y a urólogos expertos en este procedimiento. Se consideró la expertiz del participante basándose en la curva de aprendizaje para ureteroscopía flexible, que establece en 60 el número de procedimientos necesarios para completarla ${ }^{5-7}$. Por medio de correo electrónico, fueron invitados a participar en el estudio residentes de urología de diversas universidades del país y con diferentes años de formación, así como también a urólogos expertos que se desempeñan en distintos centros asistenciales. Quienes aceptaron participar, fueron citados al centro de simulación clínica de la Pontificia Universidad Católica de Chile, donde se midieron sus habilidades utilizando el modelo de simulación previamente desarrollado. Los participantes fueron separados en 3 grupos: grupo 1 (G1) para residentes sin experiencia en ureteroscopía flexible, grupo 2 (G2) para residentes con experiencia variable en este procedimiento, pero que realizaron menos de 60 ureteroscopías en el último año, y grupo 3 (G3) para urólogos expertos, los cuales efectuaron al menos 60 procedimientos en los últimos 12 meses.

A la totalidad de los participantes se les realizó de manera presencial una introducción, en la cual se les presentó el simulador desarrollado y se les explicó el objetivo de este. Posteriormente, se les solicitó realizar una navegación completa del modelo utilizando un ureterorrenoscopio flexible desechable digital (Uscope 3320, Pusen ${ }^{\mathrm{TM}}$ ). Los participantes contaban con una urografía del modelo al momento de desarrollar la exploración para guiarse en la anatomía de este (Figura 2). El tiempo máximo de navegación fue establecido en 600 segundos, considerando un tiempo suficiente para realizar la navegación del sistema colector. La medición del tiempo se realizó utilizando un cronómetro, el cual se iniciaba en el momento en que el participante ingresaba con el ureterorrenoscopio a través de la vaina de acceso ureteral, y se detenía cuando el participante verbalizaba haber encontrado todas las marcas, es decir, haber explorado todos los cálices. 
ARTÍCULO ORIGINAL
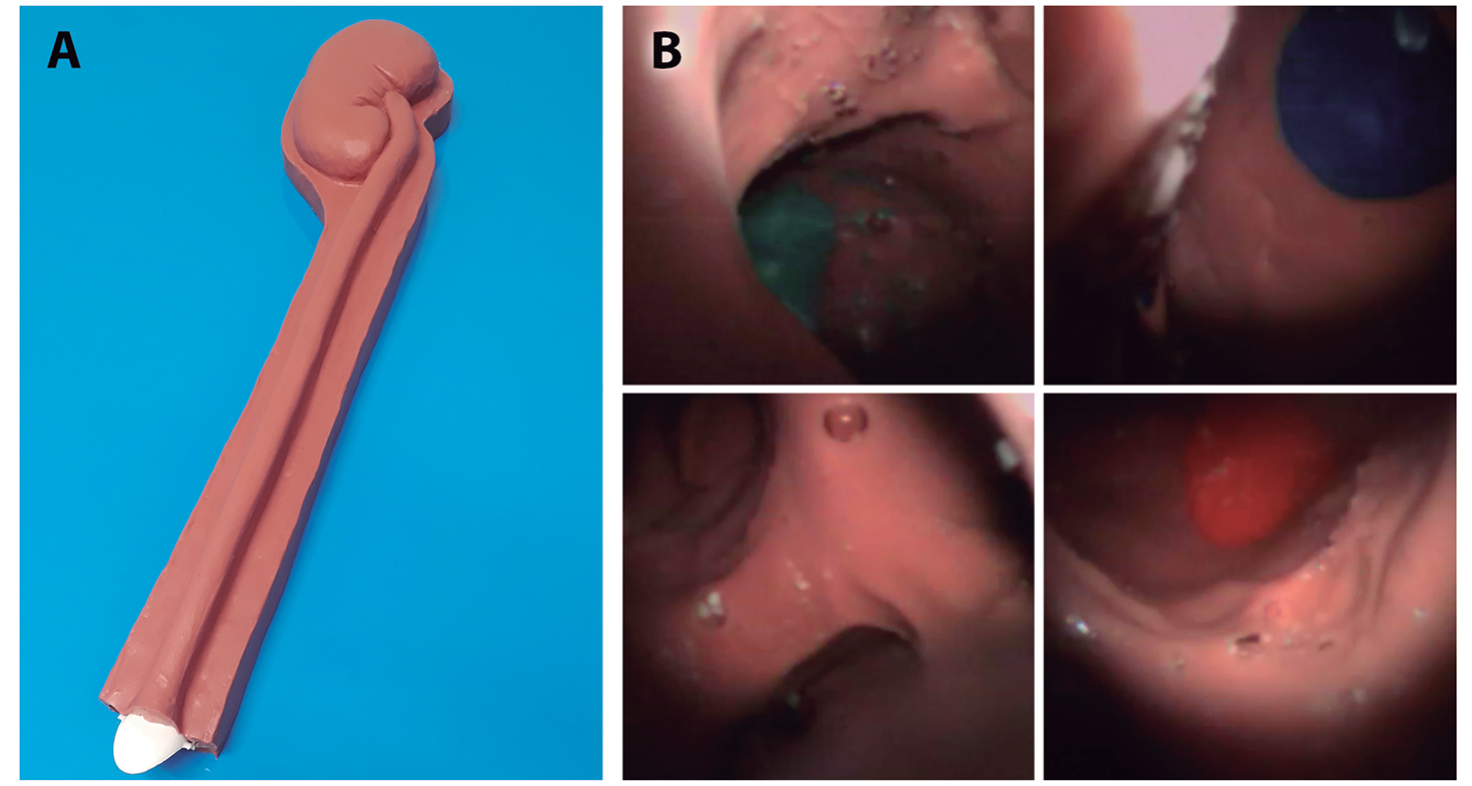

Figura 1. A: Modelo de ureterorrenoscopía flexible. B: Visión endoscópica del modelo, evidenciando la anatomía interna y las marcas en los cálices.

Además, en caso de no haber encontrado todas las marcas una vez finalizado el tiempo establecido, también detenía la medición.

Al finalizar el procedimiento, cada uno de los participantes debía completar una encuesta utilizando una escala de Likert de 10 puntos, donde $1=$ Nada Realista y $10=$ Muy Realista para validez aparente, y $1=$ Sin Utilidad y $10=$ Muy Útil para validez de contenido. Además, se preguntó a los participantes si ellos consideraban que el modelo presentaba algún problema para su uso en entrenamiento en habilidades endourológicas, solicitando, además, posibles sugerencias que podrían mejorarlo. Para la validez aparente, que corresponde a la evaluación realizada por personas no expertas acerca del realismo del modelo, se consideró las respuestas de G2 y G3; para la validez de contenido solo se consideró las respuestas de G3, ya que esta corresponde a una evaluación de la utilidad hecha por expertos $^{8,9}$. Para la validación de constructo, se midió el intervalo de tiempo total, número de marcas encontradas y la cantidad de veces de reexploración de los cálices, comparando los resultados obtenidos por los distintos grupos.

El análisis estadístico se realizó utilizando el software Stata 12.0. La evaluación de los resultados de las mediciones entre ambos grupos se llevó a cabo mediante la comparación de los promedios obtenidos, utilizando el test no paramétrico de Kruskal-Wallis.

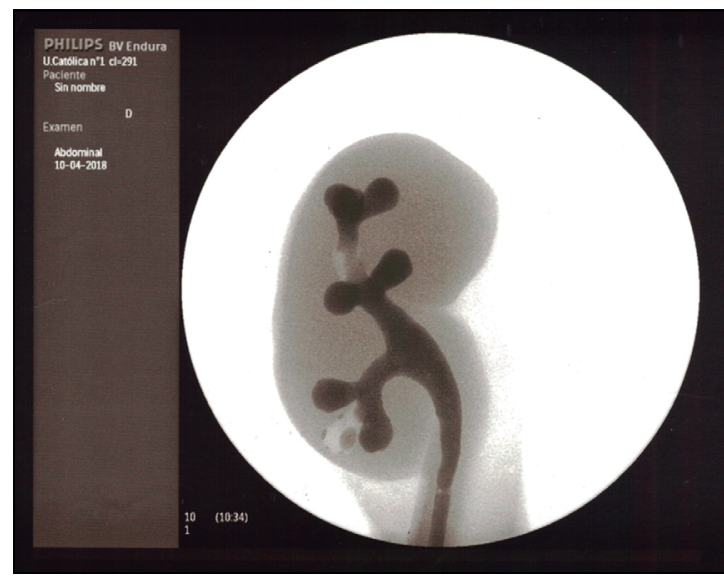

Figura 2. Urografía del modelo.

\section{Resultados}

Para el estudio, se reclutó a un total de 12 residentes de urología y 3 urólogos. Según su experiencia en ureteroscopía flexible, 6 residentes fueron asignados a $\mathrm{G} 1$, otros 6 residentes a $\mathrm{G} 2$ y 3 urólogos expertos a G3. Los datos demográficos y de experiencia en distintos procedimientos de los participantes se resumen en la Tabla 1.

\section{Validez aparente y de contenido}

Se obtuvo una mediana de 8,6 sobre 10 puntos para la utilidad del modelo como herramienta de 


\section{ARTíCULO ORIGINAL}

Tabla 1. Datos demográficos y experiencia de los participantes (promedios)

\begin{tabular}{|lccc|}
\hline & G1 & G2 & G3 \\
\hline Edad (años) & 28,8 & 30,1 & 41,3 \\
Género masculino/femenino (\%) & $66,6 / 33,3$ & $100 / 0$ & $100 / 0$ \\
Ureteroscopía (casos) & 0 & 21,6 & 105 \\
\hline
\end{tabular}

entrenamiento de habilidades en ureteroscopía flexible, y 7,5 sobre 10 puntos para el realismo de este. Los puntos a evaluar y sus respectivos resultados se pueden ver en la Tabla 2.

\section{Validez de constructo}

Al comparar los resultados obtenidos por los distintos grupos, se obtuvo que los tiempos totales de navegación fueron 504, 293 y 133 segundos para $\mathrm{G} 1, \mathrm{G} 2$ y G3 respectivamente $(\mathrm{p}=0,02)$. Por otra parte, se encontraron en promedio $5,1,6,6$ y 7,3 marcas de colores $(\mathrm{p}=0,18)$, de las 8 que tenía el modelo, presentando, además, un promedio de 95 , 3,8 y 1,3 reintentos $(\mathrm{p}=0,11)$ en la exploración de los cálices en los respectivos grupos.

\section{Discusión}

En la actualidad, las oportunidades de aprendizaje para los residentes de urología se encuentran limitadas, dificultando la adquisición de habilidades quirúrgicas en los programas de residencia ${ }^{10,11}$. Esto se debe a múltiples causas, entre ellas están la necesidad de brindar una atención segura y de calidad al paciente, las restricciones en los horarios de trabajo de los residentes, los altos costos institucionales que implican la formación quirúrgica y el surgimiento de nuevas tecnologías ${ }^{12,13}$. Además, se suma el hecho de que la curva de aprendizaje de estos procedimientos es a menudo bastante prolongada, requiriendo muchas horas de formación para perfeccionar estas habilidades ${ }^{10}$. Es así como encontramos, por ejemplo, una curva de aprendizaje que alcanza los 200 a 250 casos para una prostatectomía radical laparoscópica, hasta 105 casos para una nefrelitectomía percutánea, y de 50 a 60 casos para realizar una ureteroscopía ${ }^{5-7}$. En base a esta información establecimos el criterio de selección para agrupar a los participantes en diferentes grupos de experiencia en este estudio.

La simulación quirúrgica surge como una herramienta que otorga una nueva estrategia para la

Tabla 2. Encuesta realizada a los participantes para evaluar realismo y utilidad del modelo de simulación. Se muestran los resultados obtenidos por los expertos

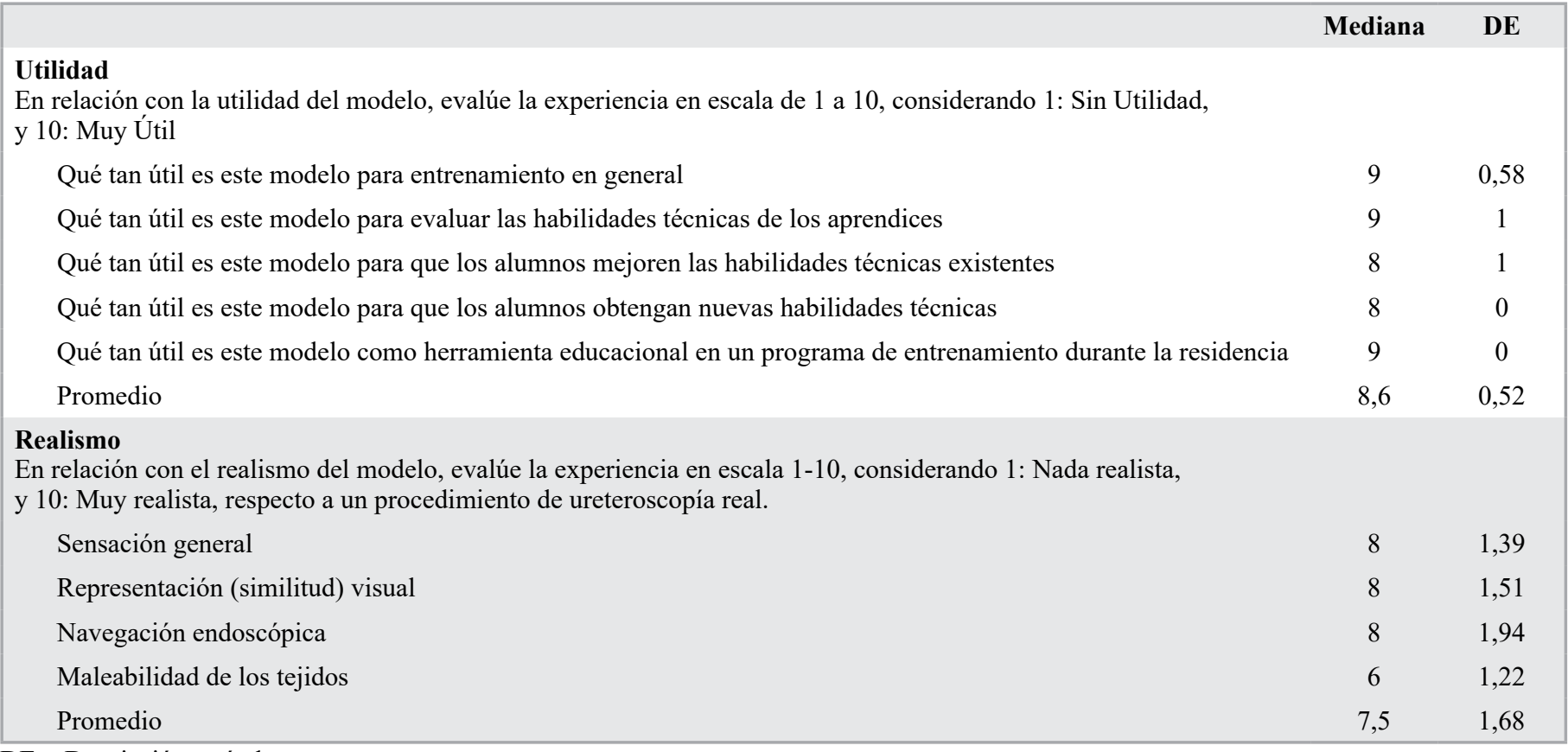

$\mathrm{DE}=$ Desviación estándar. 
adquisición de habilidades, en un entorno seguro y reproducible ${ }^{8}$. Múltiples estudios avalan la utilidad que tienen los modelos de simulación en las etapas iniciales de la curva de aprendizaje, donde los modelos de baja fidelidad demuestran ser tan efectivos como los de alta fidelidad, a un costo más bajo ${ }^{14,15}$. Además, existe evidencia creciente respecto a su utilidad en la transferencia de las habilidades obtenidas mediante simulación a cirugías reales. Una publicación del año 2016 realizada por Aloosh et al., concluyó que las habilidades obtenidas en un simulador de ureterolitectomía endoscópica (UroMentor $^{\mathrm{TM}}$ ) podían ser transferidas a cirugías reales, estableciendo la curva de aprendizaje para lograr sus objetivos en 7 intentos ${ }^{16}$.

Todo lo anterior implica la necesidad de que los simuladores quirúrgicos se encuentren debidamente validados para ser implementados en los programas de residencia ${ }^{2}$. En nuestro estudio se evaluaron tres tipos de validez, los cuales ya se encuentran previamente estandarizados para los simuladores quirúrgi$\cos ^{17}$. Es así como se evaluó la validez aparente, que corresponde a una valoración subjetiva del realismo del modelo por parte de personas no expertas, y que responde a la pregunta si el simulador representa lo que debe representar. Por otra parte, la validez de contenido, que también es una evaluación subjetiva pero realizada por expertos acerca de la utilidad del modelo como herramienta de capacitación, responde a la pregunta si el simulador enseña en la práctica lo que debe enseñar. Por último, se evaluó la validez de constructo, que corresponde a una evaluación objetiva, donde se valora la capacidad del modelo para distinguir entre individuos o grupos con diferentes niveles de experiencia ${ }^{8,9}$. De esta forma, se logró establecer validez aparente por medio de la encuesta que respondieron los participantes, evaluando el realismo del modelo con un puntaje de 7,5 en la escala de Likert de 10 puntos. En este ámbito, las principales fortalezas del modelo, y en consecuencia los puntajes más altos, se lograron respecto a la sensación general al utilizarlo y a su similitud con la realidad desde el punto de vista visual. De la misma forma, también se estableció validez de contenido, con un puntaje de 8,6, obteniendo las mejores evaluaciones respecto a su utilidad como herramienta de entrenamiento para ureterorrenoscopía en general y para evaluar habilidades en principiantes.

Finalmente, al comparar el desempeño de los 3 grupos, se logró demostrar la utilidad del modelo para distinguir entre novatos y expertos respecto a la reducción del tiempo total de navegación, los cuales fueron significativamente menores en el grupo de expertos. Sin embargo, pese a que no hubo diferencia estadísticamente significativa respecto a la cantidad de marcas encontradas, se puede observar la tendencia al aumento en éstas a medida que aumenta la experiencia; de igual forma se puede ver una disminución en cuanto a la cantidad de reintentos de exploración de los cálices en los más expertos, aun sin haber diferencias estadísticamente significativas. Esto puede deberse al número de participantes relativamente pequeño que tiene nuestro estudio, así como también puede ser a causa de una prolongación excesiva en los tiempos de navegación en personas con menor experiencia.

Al momento de preguntar a los participantes por problemas o sugerencias respecto al uso del simulador, los participantes con mayor experiencia sugerían modificar la anatomía del sistema colector, rotándolo, ya que el cáliz medio se encontraba hacia posterior en lugar de estar hacia lateral. También incluían como sugerencia el uso de irrigación. Esto concuerda con los resultados obtenidos en la encuesta respecto al realismo del modelo, donde los puntajes más bajos tenían relación con la navegación y la maleabilidad de los tejidos. Considerando que la anatomía del sistema colector de nuestro modelo se puede modificar seleccionando el UtoTac de otro paciente como modelo para imprimir, es posible ajustarse a las sugerencias de los expertos, lo que probablemente mejoraría la experiencia de simulación. En ese sentido, existe la posibilidad de desarrollar nuevos modelos con estas características, y con distintos niveles de complejidad, para su posterior validación e implementación en programas de residencia.

Dentro de las limitaciones de nuestro estudio, está la determinación del tiempo máximo establecido para poder llevar a cabo el procedimiento, el cual se definió arbitrariamente, ya que no encontramos evidencia que tomara en cuenta solo los tiempos de exploración del sistema colector en ureteroscopía flexible, sino que se consideraban los tiempos operatorios totales. Considerando que debe existir un tiempo límite, creemos que 10 minutos es un tiempo suficiente como para permitir que los principiantes realizaran una exploración completa del modelo y que en caso de no lograrlo dentro de este periodo, era poco probable que lo hicieran en un tiempo más largo. Por otra parte, para medir a los participantes en sus habilidades, no se utilizó un instrumento previamente validado, como lo es la Escala de Evaluación Global Ureteral, ya que las características actuales del modelo no lo permitían (se podrían aplicar solo 2 de los 7 puntos que posee $)^{18}$. Sin embargo, nos basamos en ítems de esta escala, seleccionando los puntos que evaluaban de forma 
objetiva las habilidades de los participantes, como lo son el tiempo, la cantidad de marcas y el número de reintento de exploración, con el fin de evitar los sesgos de medición.

Creemos que futuros estudios utilizando escalas de evaluación previamente validadas, y mejorando aquellos aspectos señalados por nuestros expertos, nos permitirán validar de constructo nuestro modelo de ureteroscopía, permitiendo el entrenamiento de residentes utilizando una herramienta útil, con un bajo costo y de fácil implementación en los programas de formación.

\section{Conclusiones}

Desarrollamos un modelo inanimado de entrenamiento mediante simulación para ureterorrenoscopía flexible, para el cual se logró establecer validez aparente y de contenido. El programa de simulación establecido, permite diferenciar novatos de expertos respecto a reducción en el tiempo de navegación. Debido a las características que posee nuestro modelo, es posible perfeccionarlo en un proceso de bajo costo, para continuar desarrollando nuevos y más complejos instrumentos de formación médica.

\section{Responsabilidades éticas}

Protección de personas y animales. Los autores declaran que para esta investigación no se han realizado experimentos en seres humanos ni en animales.

Confidencialidad de los datos. Los autores declaran que en este artículo no aparecen datos de pacientes.

Conflictos de interés: no hay.

\section{Bibliografía}

1. Brewin J, Ahmed K, Challacombe B. An update and review of simulation in urological training. International Journal of Surgery 2014;12:103-8.

2. Aydin A, Ahmed K, Shafi AMA, Khan MS, Dasgupta P. The role of simulation in urological training-A quantitative study of practice and opinions. The Surgeon 2016;14:301-7.

3. Monda SM, Weese JR, Anderson BG, Vetter JM, Venkatesh R, Du K, et al. Development and Validity of a Silicone Renal Tumor Model for Robotic Partial Nephrectomy Training. Urology 2018;114:114-20.

4. Aydin A, Shafi AMA, Shamim Khan M, Dasgupta P, Ahmed K. Current Status of Simulation and Training Models in Urological Surgery: A Systematic Review. The Journal of Urology 2016;196:312-20.

5. Brunckhorst $\mathrm{O}$, Volpe $\mathrm{A}$, van der Poel $\mathrm{H}$, Mottrie A, Ahmed K. Training, Simulation, the Learning Curve, and How to Reduce Complications in Urology. European Urology Focus 2016;2:10-8.

6. Olweny EO, Pearle MS. Update on resident training models for ureteroscopy. Curr Urol Rep. 2011;12:115-20.

7. Quirke K, Aydin A, Brunckhorst O, Bultitude M, Khan M, Dasgupta P, et al. Learning Curves of Urolithiasis Surgery: A Systematic Review. Journal of Endourology [Internet]. 24 de julio de 2018 [citado el 2 de noviembre de 2018]; Disponible en: http://www.liebertpub. com/doi/10.1089/end.2018.0425

8. Cloutier J, Traxer O. Do high-fidelity training models translate into better skill acquisition for an endourologist? Current Opinion in Urology 2015;25:143-52.

9. Schout BMA, Hendrikx AJM, Scherpbier AJJA, Bemelmans BLH. Update on Training Models in Endourology: A Qualitative Systematic Review of the Literature between January 1980 and April 2008. European Urology 2008;54:124761.

10. Wignall GR, Denstedt JD, Preminger GM, Cadeddu JA, Pearle MS, Sweet RM, et al. Surgical Simulation: A Urological Perspective. The Journal of Urology 2008;179:1690-9.

11. Salvadó JA, Oyanedel F, Sepúlveda S, Toledo H, Saavedra Á, Astroza G, et al. Validation of a high-fidelity model in ureteroscopy incorporating hand motion analysis. International Urology and Nephrology 2015;47:1265-9.

12. Varas J, Mejía R, Riquelme A, Maluenda F, Buckel E, Salinas J, et al. Significant transfer of surgical skills obtained with an advanced laparoscopic training program to a laparoscopic jejunojejunostomy in a live porcine model: feasibility of learning advanced laparoscopy in a general surgery residency. Surgical Endoscopy 2012;26:3486-94.

13. Aggarwal R, Mytton OT, Derbrew M, Hananel D, Heydenburg M, Issenberg B, et al. Training and simulation for patient safety. Quality and Safety in Health Care. 1 de agosto de 2010;19(Suppl 2):i34-43.

14. Singh A. Simulation-based training in laparoscopic urology - Pros and cons. Indian Journal of Urology 2018;34:245.

15. Matsumoto ED, Hamstra SJ, Radomski SB, Cusimano MD. The effect of bench model fidelity on endourological skills: a randomized controlled study. J Urol. 2002;167:1243-7.

16. Aloosh M, Noureldin YA, Andonian S. Transfer of Flexible Ureteroscopic StoneExtraction Skill from a Virtual Reality Simulator to the Operating Theatre: A Pilot Study. Journal of Endourology 2016;30:1120-5.

17. McDougall EM. Validation of surgical simulators. J Endourol. 2007;21:244-7.

18. White MA, DeHaan AP, Stephens DD, Maes AA, Maatman TJ. Validation of a High Fidelity Adult Ureteroscopy and Renoscopy Simulator. The Journal of Urology 2010;183:673-7. 